\title{
Análisis de la violencia en las instituciones de nivel superior y su relación con el rendimiento académico, desde un enfoque transcomplejo.
}

\section{Analysis of violence in higher-level institutions and its relationship with academic performance, from a cross-complex approach.}

\author{
Hugo A. Buitrón-Ramírez ${ }^{a}$, José F. Hernández - Gracia $^{b}$, Thelma T. Altamirano - Cardozo $^{c}$, \\ Venancio Avendaño-Hernández ${ }^{d}$
}

\begin{abstract}
:
Violence is a growing problem in educational institutions worldwide and has generated great interest in the field of educational research in recent decades. However, in Mexico there are very few studies that focus on this problem in Higher Education Institutions (IES), since most of the available studies focus mainly on basic education schools, where it is considered that the early detection of Violent behavior in students can prevent acts of abuse at secondary and higher education levels. Therefore, one of the objectives of this analysis is to know the real magnitude of the problem of violence in higher-level students and its possible relationship with academic performance, as well as the increase in violent acts where different educational actors have participated.
\end{abstract}

Keywords:

Higher education, Violence, Transcomplexity.

Resumen:

La violencia es un problema creciente en las instituciones educativas a nivel mundial y ha generado gran interés en el campo de la investigación educativa en las últimas décadas. Sin embargo en México son muy pocos los trabajos que se enfocan en esta problemática en las Instituciones de Educación Superior (IES), ya que la mayoría de estudios disponibles se enfocan principalmente a escuelas de educación básica, en donde se considera que la detección temprana de comportamientos violentos en los alumnos puede prevenir actos de maltrato en niveles de educación media y superior. Por tanto, uno de los objetivos de este análisis es conocer la magnitud real del problema de la violencia en los estudiantes de nivel superior y su posible relación con el rendimiento académico, así como el incremento de los actos violentos en donde los distintos actores educativos han participado.

Palabras Clave:

Educación superior, Violencia, Transcomplejia.

La violencia entre estudiantes es un fenómeno con antecedentes históricos que van desde la creación de los sistemas educacionales institucionalizados por grupos, hasta el día de hoy (Smokowski y Kopasz, 2005). El hecho de que determinados estudiantes sean objeto de la violencia, agresiones y el hostigamiento de otros estudiantes se describe en la literatura de la psicología de la educación (Swain, 1998) y entre otros hallazgos incluye la sugerencia que algunos adultos lo han experimentado personalmente en su trayectoria como estudiantes.
Aunque la población académica se encuentra familiarizada con el "problema del agresor y la victima" (Tapper y Boulton, 2005), mencionan que no se habían realizado esfuerzos para el estudio sistemático de la violencia intraescolar, hasta hace relativamente poco tiempo -a principios de la década de los setenta- (Olweus, 1983). Durante un par de décadas, estos intentos estuvieron limitados a revisiones críticas en la región Escandinava. Sin embargo, a finales de la década de los ochenta y principios de los noventa, el fenómeno de violencia en las instituciones educativas atrajo cierta

a Autor de Correspondencia, Universidad Tecnológica de Tula Tepeji, https://orcid.org/0000-0003-2796-1349 Email: hugoarmando.buitron@uttt.edu.mx b Universidad Tecnológica de Tula Tepeji, https://orcid.org/0000-0001-5712-3018, Email: josefrancisco.hernandez@uttt.edu.mx c Universidad Tecnológica de Tula Tepeji, https://orcid.org/0000-0003-3901-9959, Email: thelmateresa.altamirano@uttt.edu.mx d Universidad Tecnológica de Tula Tepeji, https://orcid.org/0000-0001-5577-5610, Email: venancio.avendano@uttt.edu.mx 
atención pública y de la investigación en otros países, como Japón, Inglaterra, Países Bajos, Canadá, Estados Unidos y Australia (Olweus, 1998).

Como ya se mencionó, el interés en la violencia en las instituciones educativas como tema de investigación formal, inició en la década de los 70's; teniendo como pionero a Dan Olweus, quien realizó estudios sistemáticos sobre la incidencia de la violencia entre alumnos en Noruega y Suecia en 1973 y definió por primera vez el término "bullying", que hoy conocemos como acoso escolar (Salgado, 2012).

De igual forma, Paredes, Álvarez, Lega y Vernon (2008), mencionan que a partir de los estudios de Olweus, la presencia de violencia en las instituciones educativas fue documentada en diversos países como Inglaterra, Estados Unidos, Holanda, Escocia, Irlanda, Italia, Australia y Japón. Posteriormente, Olweus realizó diversos estudios acerca de las causas y las consecuencias de ese fenómeno; enfocándose en el impacto emocional del mismo en las víctimas de violencia; teniendo como resultado el desarrollo de un programa anti-acoso implantado en Noruega.

A finales del siglo XX, el estudio de la violencia en las instituciones educativas se ha extendido debido a un incremento en los casos donde las víctimas son afectadas severamente por este fenómeno. Como ejemplo tenemos los suicidios de jóvenes que fueron víctimas de violencia escolar y acoso en países como Estados Unidos (el caso de la preparatoria Colombine en Colorado, en 1999) y España (el caso en Hondarribia, en 2004), por mencionar algunos.

En la actualidad, siguen aumentando el número de investigaciones sobre violencia en las instituciones educativas a nivel mundial, por lo cual se encuentran trabajos en los distintitos niveles educativos y en diversos países europeos, así como de Australia, Nueva Zelanda, China e Israel (Salgado, 2012). A partir de estos estudios, se puede observar que la violencia en las instituciones educativas se presenta en diferentes contextos culturales y sociales, en donde el porcentaje de alumnos implicados en estos actos oscila entre el 3 y $15 \%$ para los agresores y del 2 al 10\% para las víctimas (Moreno, 2013).

En México, de acuerdo con la Secretaría de Educación Pública, más del $70 \%$ de los alumnos de nivel básico y medio básico han sido objeto de actos de violencia por parte de sus compañeros de la escuela (Moreno, 2013). Sin embargo, los estudios que analizan los fenómenos de violencia y acoso escolar son pocos y se limitan a ciertas regiones: por ejemplo, en Guadalajara encontramos el trabajo de Vega, López, González, Valle, Flores, Vega y
López (2013), en el cual se determinó una incidencia del $17 \%$ de víctimas de maltrato escolar entre alumnos de nivel medio básico. En la Cd. De Tijuana, López, Bañuelos, Álvarez, Villalpando, Torres, Guzmán y Castillo (2012), encontraron que el $20.2 \%$ de los estudiantes se identifican como víctimas de violencia y acoso escolar, mientras que el $26.3 \%$ se reconocen como ofensores, el $44.1 \%$ como testigos, el $11.9 \%$ como cómplices y el $27.1 \%$ reportan no haber visto violencia escolar. De igual forma, las entidades de Sinaloa, Chihuahua y Jalisco, de acuerdo con la Asociación Jalisciense de Padres de Familia en Contra de la Violencia y el Acoso Escolar en la Educación (ASJAPAVA), también presentan una alta incidencia de violencia y acoso escolar (Moreno, 2013). Sin embargo, cabe mencionar que los estudios anteriormente analizados ninguno se enfoca a estudiar la correlación que puede haber entre los actos de violencia intrainstitucional y el rendimiento académico.

Una vez detectada la frecuencia con la que se presenta este problema, surgen estudios que indagan más a fondo sobre las consecuencias, los factores de causalidad, el contexto familiar y social en el que se desarrolla el problema, dando como consecuencia la implementación de programas de prevención y apoyo a las víctimas. De igual forma, Pérez, Fuentes, Gázquez, Mercader, Molero y García (2011), reportan una mayor incidencia de actos violentos entre hombres a nivel de educación media básica en comparación con las mujeres; además de identificar el nivel de escolaridad de la madre o tutora como un factor influyente en los actos de violencia; presentándose más frecuentemente en los alumnos cuyas madres tienen un menor nivel educativo. Otros estudios se enfocan a estudiar la dinámica de los actos violentos, ya que no solamente el agresor y la víctima se encuentran implicados, sino que existen colaboradores del agresor, animadores, contempladores pasivos de la agresión y defensores. En otros casos, las víctimas pueden convertirse en agresores (Moreno, 2013).

Otro estudio importante en referencia al tema, es el de Marcela Román y Javier Murillo (2011), en el cual se observa que el maltrato entre escolares es una práctica común en toda América Latina. En este trabajo, los estudiantes que sufrieron violencia de sus iguales presentaron un desempeño escolar significativamente inferior al de quienes no la experimentaron. Sin embargo, cabe mencionar que México fue excluido en el estudio. Por tanto, es de suma importancia investigar no sólo la incidencia de la violencia en las instituciones educativas, sino su correlación con el rendimiento académico que constituye un indicador del nivel de eficacia en la consecución de los objetivos curriculares. 
Para Garbanzo (2014), el rendimiento académico en estudiantes universitarios es un indicador trascendental para la evaluación de la calidad educativa, se le considera como el resultado de la unión de diferentes elementos que interactúan en el desempeño de la vida académica de los estudiantes y en donde intervienen en él aspectos de orden sociodemográfico, psicosociales, pedagógicos, institucionales y socioeconómicos; entre ellos, elementos tan variados como: la motivación, la ansiedad, la autoestima, la percepción del clima académico, el entusiasmo, el personal docente y administrativo, el sentido de propósito, entre otros.

Por otra parte el análisis realizado por el Instituto Nacional para la Evaluación de la Educación (INEE) en 2007, señala que los alumnos con más bajo rendimiento escolar, son los que han participado en más actos violentos comparado con la baja participación de los alumnos con alto rendimiento académico, así como los obtienen bajas calificaciones son los que participan con mayor frecuencia en actos violentos, ya sea como agresores o agredidos (Aguilera, García, Muñoz, Abundes, Orozco y Martínez, 2007).

Otro aspecto a considerar, es que la mayoría de estos trabajos constituyen estudios exploratorios para determinar la magnitud del problema y describir las diversas formas en que se manifiestan estos actos; así como categorizar la frecuencia del problema de acuerdo a género, edad y estrato socioeconómico. Cabe señalar que existe aún un gran campo de estudio para caracterizar el fenómeno de violencia en las instituciones de educación en México, ya que, como se puede apreciar, la mayoría de estudios sobre acoso escolar se enfoca a poblaciones de nivel medio educativo (secundaria y preparatoria); posiblemente debido a que, como menciona Pérez, Fuentes, Gázquez, Mercader, Molero y García (2011), la frecuencia de los actos de violencia aumenta a medida que aumenta la edad de los alumnos. Sin embargo, (Verlinden et al, 2013) mencionan la importancia de detectar a los agresores a edad temprana para prevenir los actos violentos en niveles educativos superiores.

Esta problemática de la violencia en las instituciones educativas en México se infiere es creciente y en donde las Instituciones de Educación Superior (IES), no son ajenas a este fenómeno y algunas se han dado a la tarea de generar estrategias para prevenir, disminuir y erradicar este problema, como es el caso del Instituto Politécnico Nacional, que ha puesto en marcha el Observatorio Zona Libre de Violencia en instituciones de Educación Superior y busca la participación de las diversas IES instauradas en la Zona Metropolitana de la Ciudad de México, para erradicar cualquier forma de violencia social y de género en los espacios académicos (Montesinos y Carrillo, 2011); sin embargo, los autores sostienen que existen pocos trabajos que ofrezcan información sobre dicho fenómeno y así fomenten mejores formas de convivencia reduciendo al mínimo la manifestación de todas las formas de violencia.

Finalmente, al relacionar violencia en las instituciones educativas y el rendimiento académico, los análisis tuvieron las siguientes conclusiones: la violencia interpares es un grave problema en toda la región; los estudiantes que sufrieron violencia de sus iguales alcanzaron un desempeño en la matemática significativamente inferior de quienes no la experimentaron; en aulas con mayores episodios de violencia física o verbal los estudiantes muestran peores desempeños que en aulas con menor violencia (Román, 2011).

Con base a lo anterior citado y a las observaciones realizadas por los miembros de un equipo transdisciplinario que indaga la temática anteriormente expuesta, se asume con base a lo señalado por Crisálida Villegas (2015) que hablar de transdiciplinariedad, implica necesariamente hablar de transcomplejidad, en donde el adjetivo complejo proviene del latín plexo, complector y expresa lo que es tejido, trenzado, enlazado y en donde la complejidad no es sinónimo de completitud, ya que lleva implícita la incertidumbre, el desorden, la ambigüedad y en donde la historia de la ciencia del siglo $X X$, la complejidad emerge en diferentes campos del saber y por primera vez en la historia de la ciencia occidental, en donde fue incorporado en numerosos campos de estudio, que según Ramentol (2004), citado por Villegas (2015) menciona que “... en la investigación se ha entrado en el laberinto de complejidad política, social, económica, científica y cultural...".

Por otra parte en opinión de Laura Savino (2015), la investigación tiene como objeto profundizar el conocer del mundo desde el flujo de experiencias del investigador, que le permitan construir la realidad y generar nuevos conocimientos, respecto al incremento de síntomas de violencia y su posible correlación con el rendimiento académico de los estudiantes.

Para finalizar y después de haber realizado esta sistematización en torno a la violencia como un factor asociado al rendimiento académico, es importante señalar que dada la complejidad del tema, es posible que en las relaciones que se suelen dar entre los distintos factores, no es posible hablar de una generalización, pues los resultados podrían ser muy diferentes según el conjunto de las relaciones causales, las poblaciones que se estudien y sus contextos. 
De igual modo, se espera que los resultados que se deriven en un futuro de esta investigación, sean de trascendencia para los directivos institucionales, administrativos y profesores que evidencien la importancia de establecer políticas y programas preventivos, que mitiguen los efectos negativos de este fenómeno y que no solo se enfoquen en resolver problemas contextuales que la originan dentro de la institución, sino que debe atraerse de forma contundente a la familia, para que participe activamente en la prevención y las posibles soluciones al problema.

\section{Referencias}

Aguilera, García, M. A., Muñoz, Abundes G. y Orozco, Martínez A. (2007). Disciplina, violencia y consumo de sustancias nocivas a la salud en escuelas primarias y secundarias de México, p.p. 57-58 México: Instituto Nacional para la Evaluación de la Educación (INEE).

Dib, Chagra Adrián (2007). Introducción al Marketing, $1^{\text {a }}$ edición, Buenos Aires, Argentina: Editorial Gran Aldea Editores.

Garbanzo, Vargas Giselle María (2014). Factores asociados al rendimiento académico tomando en cuenta el nivel socioeconómico: Estudio de regresión múltiple en estudiantes universitarios. http://www.scielo.sa.cr/pdf/ree/v18n1/a06v18n1.pdf

Kerlinger, F. (1984). Investigación del comportamiento. Técnicas y metodología. México: Iberoamericana

López, Bañuelos A., A. Álvarez, N., Villalpando, A. B., Torres, V. J. J., Guzmán, P. J. M. y Castillo, S. V. (2012). Diagnóstico de prevalencia del acoso escolar (bullying) en primarias, secundarias y preparatorias del municipio de Tijuana. CETYS Universidad, Campus Tijuana. https://www.guao.org/sites/default/files/buenas\%20practicas/Dia gn $\%$ C3\%B3stico $\% 20$ de $\% 20$ prevalencia\%20del\%20acoso $\% 20$ esc olar\%20en $\% 20$ primarias $\% 2 \mathrm{C} \% 20$ secundarias $\% 20 \mathrm{y} \% 20$ preparato rias.pdf

Montesinos, Rafael y Carrillo, Rosalía (2011). El crisol de la violencia en las universidades públicas. El cotidiano 2011. http://www.redalyc.org/service/redalyc/downloadPdf/325/325209 $35006 / \mathrm{El}+$ crisol+de+la+violencia+en+las+universidades+p $\% \mathrm{FAb}$ licas/1

Moreno, González, M. G. (2013). El bullying o violencia escolar en México, el reto de la educación básica actual: reflexión y prospectiva, Cultura y Generación de Conocimiento en América Latina, p.p. 161-163, México: Universidad Pedagógica Nacional.

Olweus, D. (1983), Low achievement and aggressive behaviour in adolescent boys. En D. Magnusson y V. Allen, (editors), Human development. An international perspective. Academic Press: Nueva York.

Olweus, D. (1998). Conductas de acoso y amenazas entre estudiantes. Ediciones Morata, Madrid, España.

Paredes, M. T., Álvarez, M. C., Lega, L. I. y Vernon, A. (2008). Estudio exploratorio sobre el fenómeno del "Bullying" en la ciudad de Cali, Colombia, pp. 295-317, Revista latinoamericana ciencias sociales niñez juventud 6 (1)

Pérez, Fuentes, M. C., Gázquez, J. J., Mercader, I., Molero, M. M. y García, M. M. (2011). Rendimiento académico y conductas antisociales y delictivas en alumnos de Educación Secundaria Obligatoria, International Journal of Psychology and Psychological Therapy 11(3), pp. 401-412.

Ramentol, S. (2004). Teorías del desconcierto. España: Urano tendencias.

Román, M. y Murillo, F. J. (2011). América Latina: violencia entre estudiantes y desempeño escolar. p. 38, Revista CEPAL vol. 32.

Salgado, Lévano Ana Cecilia (2012). Revisión de las investigaciones acerca del Bullying: desafíos para su estudio. pp. 130-131, En Benítez L. y Carozzo J. (Coords.), Bullying y convivencia en la escuela. Lima: Dennis Morzán Delgado Impresiones. https://www.researchgate.net/publication/271647471_Revision_d
e_las_investigaciones_acerca_del_Bullying_Desafioso_para_su_ estudio

Savino, Laura (2015). La transcomplejidad y la producción del conocimiento transdisciplinario, Diálogos de posdoctorado Volumen I, Numero 7, Año 2015, Venezuela: Universidad Bicentenaria de Aragua.

Smokowski, P. R. and Kopasz, K. H. (2005). Bullying in school: an overview of types, effects, family characteristics, and intervention strategies. Pp. 101-110, Children and Schools, 27. https://eric.ed.gov/?id=EJ724855

Swain, J. (1998). ¿What does bullying really mean? p.p. 358-364, Educational Research, 40.

Tapper, K. and Boulton, M. J. (2005). Victim and peer group responses to different forms of aggression among primary school children. Pp. 238-253, Aggressive Behavior, 31. http://onlinelibrary.wiley.com/doi/10.1002/ab.20080/abstract

Vega, López, M. G., González, G. J., Valle, M. A., Flores, M. E., VegaLópez, A. (2013). Acoso escolar en la zona metropolitana de Guadalajara, México: prevalencia y factores asociados. https://www.scielosp.org/scielo.php?pid=S18512652013000200005\&script=sci_abstract\&tlng=es

Verlinden, M., Veenstra, R., Ringoot, A. P., Jansen, P. W., Raat, H., Hofman, A., Jaddoe, V.W.V., Verhulst, F. C., y Tiemeier, H. (2013). Detecting bullying in early elementary school with a computerized peer-nomination instrument. Psychological Assessment.

Villegas, G. Crisálida (2015). La investigación transcompleja un transparadigma, Diálogos de posdoctorado Volumen I, Numero 7, Año 2015, Venezuela: Universidad Bicentenaria de Aragua. 